\title{
HALLAZGOS CERÁMICOS Y SU CRONOLOGÍA EN EL ÁREA COMPRENDIDA ENTRE EL EXTREMO SUR DEL MACIZO DEL DESEADO Y LA CUENCA DEL RÍO SANTA CRUZ (PATAGONIA SUR, ARGENTINA)
}

\author{
NATALIA A. CIRIGLIANO ${ }^{a}$, TERESITA MONTENEGRO ${ }^{b}$, NORA V. FRANCO', \\ GEORGE A. BROOK ${ }^{\mathrm{d}} \&$ ALEXANDER CHERKINSKYe
}

\begin{abstract}
RESUMEN
El objetivo de este trabajo es informar sobre la recuperación de seis tiestos en el área ubicada entre el extremo sur del Macizo del Deseado y el río Santa Cruz. Si bien se trata de un registro arqueológico poco abundante, constituye un aporte significativo al conocimiento sobre el uso de esta tecnología entre grupos cazadores-recolectores en estas latitudes y en espacios intermedios entre la costa y la cordillera. Se presenta la información cronológica (AMS) e isotópica $\left(\delta^{13} \mathrm{C}\right.$ y $\left.\delta^{15} \mathrm{~N}\right)$ generada a partir de la datación y análisis de las sustancias adheridas a las superficies planas de dos fragmentos cerámicos. Asimismo, se exhiben los resultados de los análisis macroscópicos de los tiestos y microscópicos de las pastas. Sobre esta base se indaga acerca de las características de la cerámica, su posible función y el marco cronológico de uso de esta tecnología. Los resultados alcanzados son consistentes con aquéllos obtenidos en la región y apuntan a un uso tardío de la cerámica, a la utilización de recursos de estepa y a la posible obtención y transporte de recipientes por grupos indígenas con alta movilidad desde otros espacios.
\end{abstract}

PALABRAS CLAVE: cazadores-recolectores, cerámica, cronología, variabilidad, Patagonia sur.

\section{CERAMIC FINDINGS AND THEIR CHRONOLOGY IN THE AREA BETWEEN THE SOUTHERN DESEADO MASSIF AND THE SANTA CRUZ RIVER BASIN (SOUTHERN PATAGONIA, ARGENTINA)}

\section{ABSTRACT}

The purpose of this paper is to provide information about six sherds recovered from the area between the Southern Deseado Massif and the Santa Cruz River Basin. Very few sherds have been recovered from

a Consejo Nacional de Investigaciones Científicas y Técnicas, Instituto Multidisciplinario de Historia y Ciencias Humanas. Saavedra 15, 5to piso, C.A.B.A. (C.P. 1083), Argentina. $\measuredangle$ naticirigliano@ hotmail.com

b Universidad de Buenos Aires, Facultad de Ciencias Exactas y Naturales, IGEBA-CONICET Argentina. mon@gl.fcen.uba.ar

c Consejo Nacional de Investigaciones Científicas y Técnicas, Instituto Multidisciplinario de Historia y Ciencias Humanas/ Universidad de Buenos Aires, Facultad de Filosofía y Letras. Saavedra 15, 5to piso, C.A.B.A. (C.P. 1083), Argentina/Puán 480, C.A.B.A (C.P. 1406), Argentina. nufranco2008@gmail.com

d Department of Geography, University of Georgia, Athens, GA 30602, U.S.A. gabrook@uga.edu

e Center for Applied Isotope Studies, the University of Georgia, Athens, Georgia, GA 30602, U.S.A. acherkin@uga.edu 
this region previously, and so these findings make a significant contribution to what is known about ceramic technologies used by hunter-gatherers at these latitudes, in an area located between the Andean range and the Atlantic coast. This paper provides macroscopic and microscopic data of the six sherds, which show considerable variability. It also reports isotopic values $\left(\delta^{13} \mathrm{C}\right.$ and $\left.\delta^{15} \mathrm{~N}\right)$ and direct AMS radiocarbon ages for organic residues adhering to the flat faces of two sherds. The results are consistent with those from earlier studies indicating the use of ceramic technology by highly mobile indigenous groups in Southern Patagonia for steppe resources. Variations in sherd composition suggest that the original ceramic containers were probably acquired and transported into the region from other spaces.

KEY WORDS: hunter-gatherers, ceramic, chronology, diversity, southern Patagonia.

\section{INTRODUCCIÓN}

El registro cerámico localizado en el sur de la región patagónica y al este de la cordillera de los Andes es poco frecuente. Este fue asignado temporalmente al último tramo del Holoceno tardío mayormente por relación contextual con otros materiales y/o depósitos fechados (Menghin, 1952; Gradin, 1976; Gradin et al. 1979; Massone, 1984; Bird, 1988; Mena \& Jackson, 1991; Martinic \& Prieto, 1998; Goñi, 2000-2002; Goñi et al. 2000-2002; Cassiodoro \& Tchilinguirian, 2007; Moreno, 2009; Cassiodoro, 2010; Hammond \& Zubimendi, 2013; Zubimendi, 2015 ; entre otros) y, en menor medida, por la datación de materia orgánica adherida a la superficie de los tiestos (Cassiodoro \& Tessone, 2014; Chaile et al. 2019) o de los minerales sometidos a calentamiento (Mena \& Lucero, 2004). Las dataciones obtenidas por AMS y por termoluminiscencia al NO del área de estudio se encuentran comprendidas entre ca. 880 y 100 años AP (Mena \& Lucero, 2004; Cassiodoro \& Tessone, 2014; Chaile et al. 2019).

Los estudios más completos sobre el registro cerámico fueron desarrollados al NO de la región $e$ incluyen análisis macroscópicos, microscópicos $y$ de isótopos estables en residuos orgánicos ( $v$. Cassiodoro \& Tchilinguirian, 2007; Cassiodoro, 2008, 2009; Goñi et al. 2011-2012; Cassiodoro \& Tessone, 2014; Cassiodoro et al. 2014; Chaile et al. 2018). Por otra parte, se hicieron estudios macroscópicos sobre el material cerámico procedente de la costa norte de la provincia de Santa Cruz (Trola \& Ciampagna, 2011; Roumec et al. 2017) y se indicó su existencia y tipo de decoración al sur de los espacios mencionados (Bellelli, 1980; Massone, 1984; Gradin, 2000; entre otros).

Entre los años 2011 y 2014 se recuperaron seis fragmentos cerámicos en el área localizada entre el extremo sur del Macizo del Deseado y el río Santa Cruz, dos de los cuales registraron sustancias adheridas que fueron sometidas a dataciones radiocarbónicas y análisis isotópicos. Asimismo, se describieron las características macro-microscópicas de los tiestos con el objetivo de conocer cuál era la variabilidad que presentaban y el papel de esta tecnología en el espacio de estudio, intermedio entre la costa atlántica y la cordillera. A continuación y para contextualizar los hallazgos, se sistematizó la información arqueológica y documental de espacios cercanos al área de estudio, al este de la cordillera de los Andes, entre los ríos Deseado y Santa Cruz.

\section{ANTECEDENTES ARQUEOLÓGICOS Y DOCUMENTALES}

El uso de la tecnología cerámica entre los ríos Deseado y Santa Cruz fue estimado entre ca. 1.200 y 100 años AP -ca. 920-1.900 DC- (Gradin \& Aguerre, 1991; Gradin, 2000; Goñi, 2000; Goñi et al. 2000-2002; Moreno \& Videla, 2008; Cassiodoro, 2008; Hammond \& Zubimendi, 2013; Cassiodoro \& Tessone, 2014; Zubimendi et al. 2015; Ciampagna, 2016; entre otros). Desde el punto de vista arqueológico, la mayor densidad de tiestos se encontró al NO (Outes, 1904; Gradin, 1976; Gradin et al. 1979; García, 1987; Mengoni Goñalons, 1987; Goñi, 2000, 2000-2002; Goñi et al. 2000-2002; Cassiodoro \& Tchilinguirian, 2007; Cassiodoro, 2008; Cassiodoro \& García Guraieb, 2009; Goñi et al. 2010; Cassiodoro et al. 2013; Sacchi, 2013; Cassiodoro et al. 2014; Chaile et al. 2019; entre otros) y NE del área de estudio (Moreno \& Videla, 2008; Trola \& Ciampagna, 2011; Hammond et al. 2013; Zubimendi et al. 2015; Roumec et al. 2017; entre otros). Al sur de esos 
espacios el registro cerámico es exiguo (Verneau, 1903; Gradin \& Aguerre, 1983; Durán, 1985; Gradin, 2000; Belardi et al. 2013; entre otros). Específicamente, en el extremo sur del Macizo del Deseado, se detectaron dos sitios con esta tecnología: Zanjón de Piedra (Gradin \& Aguerre, 1983; Aguerre, 1987) y sitio Cueva Laguna Colorada (Durán, 1985). Más al sur, Verneau (1903) reconoció unos pocos tiestos en La Salina, margen norte de la cuenca inferior del río Santa Cruz (v. Bellelli, 1980), mientras que Gradin (2000) ofreció información sobre la existencia de tiestos en la Estancia La Rosita -río Chalía- y en cercanías de la localidad de Comandante Luis Piedra Buena (Fig. 1). En líneas generales y según la bibliografía mencionada, la cerámica arqueológica fue obtenida tanto en superficie como en estratigrafía y en sectores con características distintas: cotas altas y bajas, a cielo abierto y bajo roca, en cercanías de fuentes de agua -costa, márgenes de lagos y/o cañadones-, estructuras de entierros, concheros y parapetos. Según las descripciones, los tiestos registran variabilidad macroscópica. Los hay con/ sin decoración (e.g. incisa), con diversidad en cocción -reductora, oxidante, mixta-, sin tratamiento de las superficies o con superficies alisadas/pulidas, espesores diversos - $2,2 \mathrm{~mm}$ a $9 \mathrm{~mm}-$, posible forma globular o subglobular abierta y con/sin sustancias adheridas -e.g. hollin-.

Cassiodoro y Tchilinguirian (2007) y Cassiodoro (2009) realizaron análisis microscópicos $y$, sobre la base de aspectos composicionales de las pastas y arcillas del NO de la provincia de Santa Cruz, propusieron a la cuenca Salitroso-PosadasPueyrredón como una posible área de manufactura cerámica. Cabe señalar que en los sectores bajos y altos localizados al NO del área de estudio se recuperó un total de 1.099 tiestos que corresponderían a 24 contenedores (Chaile et al. 2019). En este sentido, los autores mencionan que la confección local y el uso de la cerámica se relacionarían con una baja de la movilidad residencial de grupos cazadoresrecolectores (Chaile et al. 2019).

En líneas generales, el uso de la tecnología cerámica entre grupos cazadores-recolectores con alta movilidad fue vinculado con el procesamiento de alimentos, transporte de recursos, almacenamiento de grasas, prestigio y práctica ritual (v. Gradin, 1976; Borrero, 1994-95; Cassiodoro \& Tchilinguirian,
2007; Cassiodoro et al. 2013). Cabe señalar que los resultados de los análisis de isótopos estables $\left(\delta^{13} \mathrm{C}\right.$ y $\left.\delta^{15} \mathrm{~N}\right)$ en residuos orgánicos de tiestos recuperados en proximidades del área bajo estudio fueron compatibles con el procesamiento de recursos animales y vegetales de estepa (Cassiodoro \& Tessone, 2014; Chaile et al. 2018).

Las fuentes documentales también informan sobre el uso de recipientes de barro y/o cerámicos durante el primer contacto hispano-indígena en bahía San Julián -costa atlántica- (Pigaffeta, 2001 [1899], aunque $v$. Martinic \& Prieto, 1998 sobre este tema) y durante momentos muy tardíos del siglo XX en la zona del río Pinturas (Aguerre, 2000). A su vez, a partir del año 1.670 DC las crónicas aportan datos sobre el reconocimiento de cerámica arqueológica en bahía San Julián (Narborough, 2007 [1694]), isla Pavón (Lista, 2007 [1879]), en proximidades de Spring Creek, este del lago Ghio y sudeste del lago Buenos Aires (Hatcher, 2003 [1903]) (Fig. 1).

\section{ANTECEDENTES TEÓRICOS}

La producción, uso y transporte de cerámica pueden ocurrir en sociedades cazadoras-recolectoras (Simms et al. 1997; Cornejo \& Sanhueza, 2003; Eerkens, 2003; Cassiodoro \& Tchilinguirian, 2007; Sugrañes, 2010; entre otros). Se ha señalado que la baja frecuencia de elementos cerámicos entre grupos con alta movilidad podría deberse a diversos factores, tales como los altos costos de transporte, el riesgo de fractura o la falta de tiempo suficiente para completar el ciclo de producción (Eerkens et al. 2002; Eerkens, 2003; entre otros). En este contexto, la cerámica se ha identificado en áreas donde las materias primas necesarias para su manufactura se hacen presentes y/o en espacios utilizados reiteradamente (Simms et al. 1997; Eerkens, 2003; entre otros). De esta manera, es posible tanto el transporte de vasijas como el equipamiento de determinados lugares para evitar el acarreo durante los desplazamientos (Eerkens, 2003).

Los recipientes entre grupos con alta movilidad suelen presentar tamaños pequeños, formas estandarizadas, espesores delgados, superficies alisadas y textura fina (Cornejo \& Sanhueza, 2003; Eerkens, 2003, 2008; entre otros). Estas características los harían menos pesados y más 


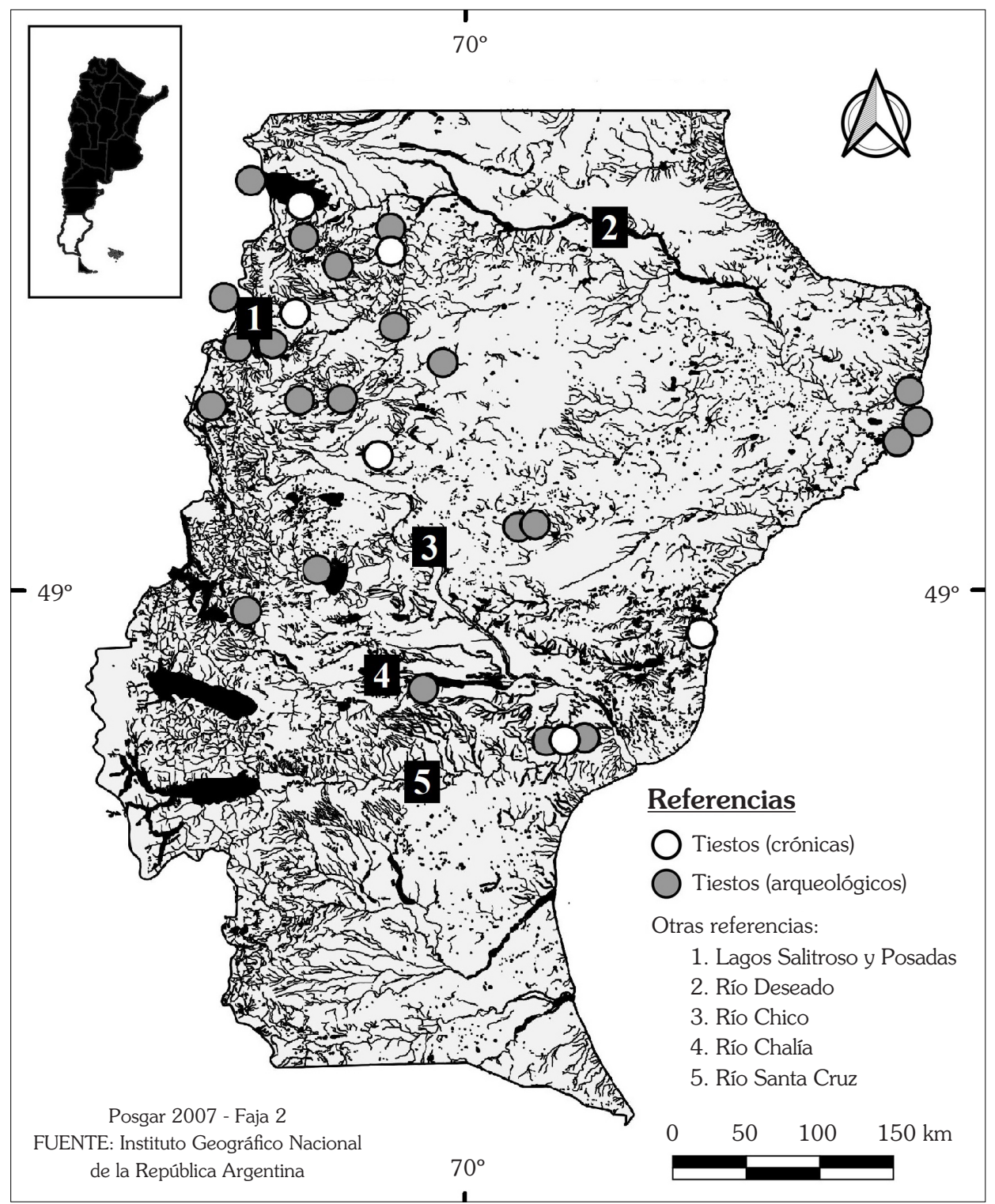

Fig. 1. Localización de la tecnología cerámica entre los ríos Deseado y Santa Cruz.

resistentes a la fractura, a la vez que contribuirían a un calentamiento más rápido, minimizando el tiempo de cocción (Kingery et al. 1976; Braun, 1983; Rice, 1987; Eerkens, 2003). Debido al riesgo de fractura de los recipientes y a las limitaciones del acarreo (e.g. Kuhn, 1994), en caso de transporte se espera baja densidad de material cerámico. Su traslado desde otros espacios y/o su obtención de otros grupos daría por resultado una alta variabilidad o diversidad cerámica -e.g. materias primas- (Simms et al. 1997; Cornejo \& Sanhueza, 2003).

\section{MATERIALES Y MÉTODOS}

El registro cerámico que se analizó en este trabajo se encuentra conformado por seis tiestos. Los fragmentos cerámicos se localizaron en el extremo sur del Macizo del Deseado $(n=1)$, curso medio del río Chico $(n=2)$, curso medio del río Chalía $(n=1)$ y curso superior del río Santa Cruz $(n=2)$ (Fig. 2). Todos los tiestos se recuperaron en posición superficial, al aire libre, en ambientes de erosión eólica y en cercanías de fuentes de agua, próximos 
a chorrillos $(n=2)$, cañadones $(n=1)$, ríos $(n=2)$ y lagunas $(n=1)$. Tres de las muestras fueron obtenidas en el marco de trabajos sistemáticos de muestreo. En uno de los casos, proviene del sitio Zanjón de Piedra, extremo sur del Macizo del Deseado, donde previamente se había recolectado también cerámica (Gradin \& Aguerre, 1983; Aguerre, 1987). Los otros dos ejemplares proceden de tareas de prospección realizadas en inmediaciones de cerro Ventana, cuenca media del río Chico (Cirigliano, 2016). Finalmente, los restantes ejemplares $(n=3)$ provienen de donaciones hechas por pobladores locales. Se trata de las muestras procedentes de las cercanías del casco de la Estancia El Tranquilo (cuenca superior del río Santa Cruz) y de la cuenca media del río Chalía, en proximidades de Estancia La Rosita, donde también Gradin (2000) había identificado su presencia (Fig. 2). Los tiestos obtenidos durante los trabajos de campo por el equipo de investigación $(n=3)$ se encontraron asociados con tecnología lítica. Se desconoce la tecnología relacionada con los fragmentos cerámicos recuperados por los pobladores del área.

Las muestras $(n=6)$ se analizaron $y$ fotografiaron en el gabinete. Los únicos dos tiestos con residuos orgánicos adheridos se enviaron al Center for Applied Isotope Studies (CAIS) de la Universidad de Georgia. Las superficies de esos tiestos se limpiaron con acetona. Para efectuar la datación por radiocarbono (AMS) se removieron con bisturí los residuos orgánicos. Éstos se encontraban sujetos a las superficies lisas, planas o ligeramente biseladas de los tiestos y no a sus bordes fracturados, lo que sugería que se hallaban presentes con anterioridad a la rotura de la pieza y, por lo tanto, podían vincularse con actividades de uso de los recipientes cerámicos. Cabe señalar que los bordes angulosos de los tiestos ofrecieron integridad a la muestra, permitiendo inferir que no ha habido un transporte significativo por agentes naturales (ver infra).

Las muestras de residuos fueron tratadas con $5 \%$ de $\mathrm{HCl}$ a $80^{\circ} \mathrm{C}$ durante una hora y luego se lavaron con agua desionizada en un filtro de fibra de vidrio y se enjuagaron con $\mathrm{NaOH}$ diluido para eliminar la posible contaminación por ácidos húmicos. Luego se trataron nuevamente con $\mathrm{HCl}$ diluido, se lavaron con agua desionizada y se secaron a $60^{\circ} \mathrm{C}$. Las muestras limpias se quemaron a $900^{\circ} \mathrm{C}$ en ampollas de cuarzo selladas en presencia de
CuO. El dióxido de carbono resultante se purificó criogénicamente a partir de los otros productos de reacción y se convirtió catalíticamente en grafito usando el método de Vogel et al. (1984). Las relaciones de grafito ${ }^{14} \mathrm{C} /{ }^{13} \mathrm{C}$ se midieron utilizando el espectrómetro de masas con acelerador CAIS 0,5 MeV. Los valores obtenidos se compararon con aquéllos proporcionados por Oxalic Acid I (NBS SRM 4990). Las proporciones de ${ }^{13} \mathrm{C} /{ }^{12} \mathrm{C}$ se midieron por separado usando un espectrómetro de masas de relaciones isotópicas estables y se expresaron como $\delta^{13} \mathrm{C}$ con respecto a VPDB, con un error de menos de 0,1\%o.

Las fechas no calibradas se encuentran en años radiocarbónicos (antes de 1.950 años AP), usando la vida media de ${ }^{14} \mathrm{C}$ de 5.568 años, y se corrigieron para el fraccionamiento isotópico en $\delta^{13} \mathrm{C}(-25 \%$ \%). El error es una desviación estándar y refleja las incertidumbres estadísticas y experimentales. Las edades de radiocarbono se calibraron en el nivel de probabilidad $2 \sigma$ utilizando CALIB 7.1 html (Stuiver \& Reimer, 1993) y la curva de calibración atmosférica del hemisferio sur (SHCal13) de Hogg et al. (2013).

Los análisis macroscópicos de los tiestos y microscópicos de las pastas siguieron los lineamientos metodológicos que se encuentran en la Primera Convención Nacional de Antropología (1966), Balfet et al. (1992), Orton et al. (1997), Solá (2001, 2011), Frére et al. (2012), entre otros. Los análisis macroscópicos y morfológicos contemplaron las siguientes variables: 1) forma; 2) tamaño de los tiestos -ancho y longitud en $\mathrm{mm}$-; 3) grosor de las paredes en $\mathrm{mm}$-variable que ha sido medida preferencialmente en los sectores de la pieza que no presentaban descascaramiento de las superficies-. Se utilizó el criterio de clasificación de Frére et al. (2012) para espesores delgados $(<5,2$ $\mathrm{mm}$ ), medianos (entre 5,2 y $8 \mathrm{~mm}$ ) y gruesos ( $>8$ $\mathrm{mm}$ ); 4) acabado de las superficies; 5) decoración; 6) color (Munsell Soil Color Chart, 1992); y 7) estado de los bordes -e.g. angulosos/redondeados-. Esta última variable permitió inferir si existió algún tipo de agente natural -acción del agua o vientovinculado con el transporte de los tiestos y/o su exposición a la intemperie (Powers, 1953; Skibo, 1987; Waters, 1992). Asimismo, se realizaron cortes delgados de todos los tiestos y se procedió a la observación microscópica y descripción 
de las secciones. Se utilizó un microscopio de polarización petrográfico con platina giratoria y binoculares LEITZ WETZLAR con distintos aumentos $-3,5 \mathrm{x}, 10 \mathrm{x}$ y $25 \mathrm{x}^{-}$, consignándose la presencia de inclusiones, matriz y poros. Se siguió el criterio de Giusta (2017) para la clasificación de la densidad de poros o cavidades. En este sentido, se consignó la densidad de poros como alta (> $15 \%)$, moderada (entre 5 y 15\%) y baja $(<5 \%)$. Se describió el porcentaje de inclusiones por medio
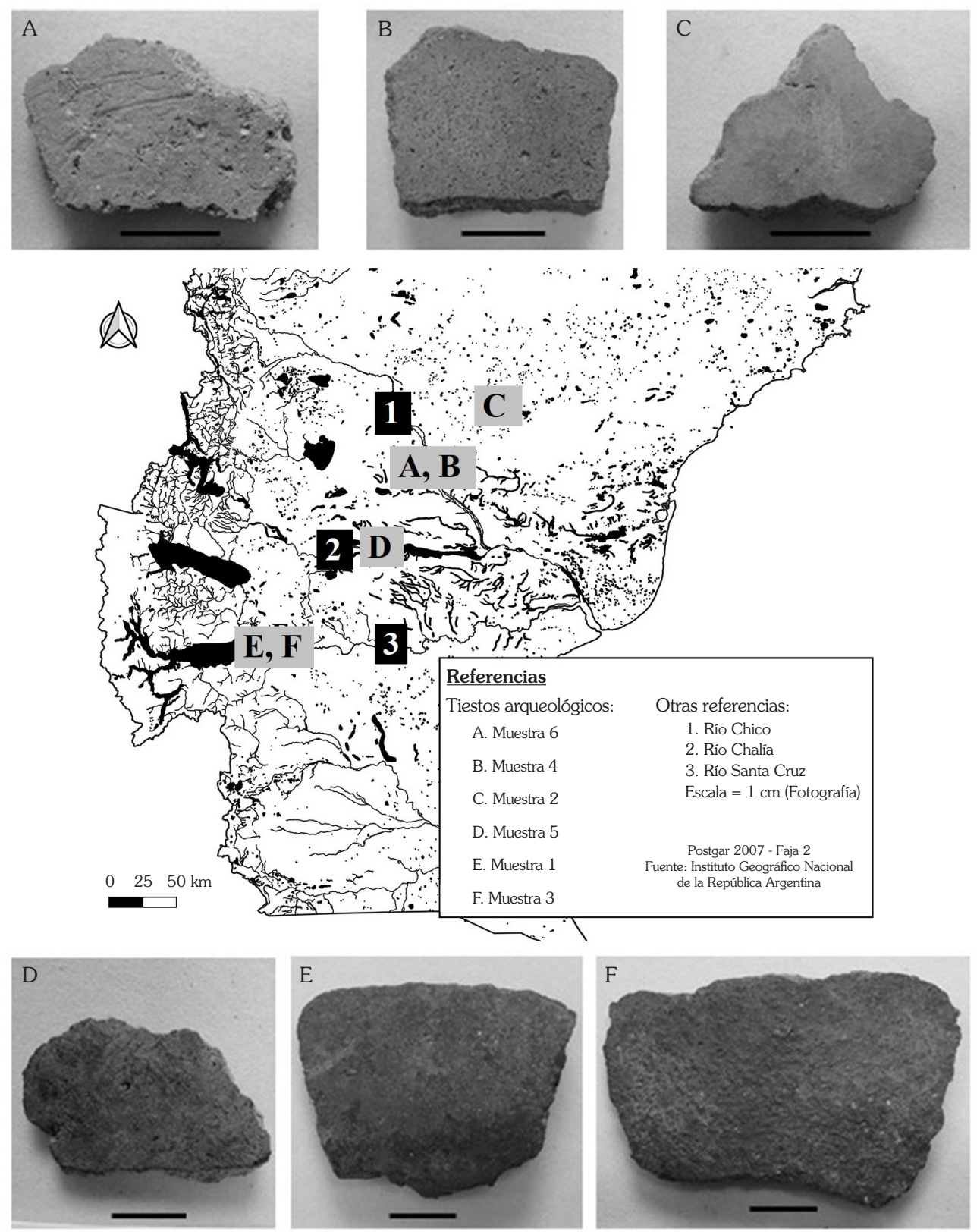

Fig. 2. Localización de los tiestos recuperados en el área de estudio.

Referencias: A y B. cerro Ventana (curso medio, río Chico); C. extremo sur del Macizo del Deseado (v. sitio Zanjón de Piedra, Aguerre, 1987); D. cuenca media del río Chalía, proximidades de Ea. La Rosita; E y F. Ea. El Tranquilo (curso superior, río Santa Cruz). Las referencias D y C se superponen con hallazgos arqueológicos previos realizados por otros equipos de investigación. 
de cuadros visuales que estiman proporciones, la composición mineralógica de la pasta, y la forma y tamaño máximo y mínimo de las inclusiones (sensu Escala Udden-Wentworth en Folk, 1974).

Los resultados isotópicos fueron comparados con aquéllos obtenidos por investigadores de áreas cercanas (Tessone \& Panarello, 2008-2010; Cassiodoro \& Tessone, 2014; Chaile et al. 2018) y carbones arqueológicos de las cuencas de los ríos Chico y Santa Cruz (Brook et al. 2018).

\section{RESULTADOS}

\section{Análisis macroscópicos}

Los tiestos recuperados entre el extremo sur del Macizo del Deseado y el río Santa Cruz fueron asignados como fragmentos de cuerpo.
Los espesores son medianos $(83,33 \%)$ y delgados $(16,67 \%)$ y están comprendidos entre los 4 y 8 mm (Tabla 1). Ningún tiesto registra decoración. El $66,66 \%$ de los fragmentos está alisado en ambas caras, aunque existen distintos grados en la calidad del tratamiento. Un tiesto posee pulido y falso engobe (16,67\%) (sensu Solá, 2001) y otro, una superficie tosca -cara interna- $(16,67 \%)$ e indeterminada -cara externa-. Esta última es áspera al tacto como consecuencia de la acción de agentes erosivos. Todos los ejemplares presentan fractura irregular y bordes mayormente angulosos (Powers, 1953). Se ha señalado que a mayor angulosidad, menor desgaste y/o transporte (Solá, 2001).

A partir del color de los cortes delgados se puede inferir la existencia de cocción oxidante $\mathrm{u}$ oxidante incompleta (Tabla 2) (v. Balfet et al. 1992; Orton et al. 1997; Solá, 2001). Cinco

Tabla 1. Dimensiones y tratamiento de la superficie de los tiestos.

\begin{tabular}{cccccc}
\hline & & & & \multicolumn{2}{c}{ Tratamiento de superficie } \\
\hline Muestra & Procedencia & Dimensión $(\mathrm{mm})$ & Espesor $(\mathrm{mm})$ & externo & Anterno \\
\hline 1 & $\begin{array}{c}\text { Río Santa Cruz, } \\
\text { curso superior }\end{array}$ & $32,5 \times 42$ & 8 & Alisado & Alisado \\
2 & $\begin{array}{c}\text { Extremo sur del Macizo } \\
\text { del Deseado }\end{array}$ & $20 \times 25,5$ & 6 & Pulido, falso engobe & Pulido, falso engobe \\
3 & Río Santa Cruz, curso & $36,5 \times 55,5$ & 26,5 & Indeterminado & por erosión \\
4 & Río Chico, curso medio & $22,5 \times 25$ & 6,5 & Alisado & Alisado \\
5 & Río Chalía, curso medio & $20 \times 31,5$ & 4 & Alisado & Alisado \\
6 & Río Chico, curso medio & $18 \times 28$ & 5,5 & & Alisado \\
\hline
\end{tabular}

Tabla 2. Color de las superficies del tiesto y del corte delgado (Munsell Soil Color Chart, 1992).

\begin{tabular}{|c|c|c|c|}
\hline Muestra & Superficie externa & Superficie interna & Corte delgado \\
\hline \multirow{2}{*}{1} & $10 Y R 3 / 2$ & 10YR 3/1 & 10YR 2/2 (very dark brown) y \\
\hline & (very dark grayish brown) & (very dark gray) & 10YR 3/6 (dark yellowish brown) \\
\hline \multirow{2}{*}{2} & $10 Y R 5 / 4$ & $10 Y R 5 / 3$ & 5YR 5/8 (yellowish red) \\
\hline & (yellowish brown) & (brown) & Variación de la tonalidad \\
\hline \multirow{2}{*}{3} & $2.5 Y 3 / 1$ & $2.5 Y 4 / 1$ & 10R 5/1 (reddish gray) y \\
\hline & (very dark gray) & (dark gray) & 5YR 3/3 (dark reddish brown) \\
\hline \multirow{2}{*}{4} & $10 Y R 5 / 4$ & 10YR 5/1 (gray) y & 10YR 4/6 (dark yellowish brown) \\
\hline & (yellowish brown) & $2.5 Y 5 / 3$ (light olive brown) & Variación de la tonalidad \\
\hline \multirow{2}{*}{5} & $2.5 Y 2,5 / 1$ & $2.5 Y 5 / 2$ & 7.5YR 2,5/3 (very dark brown) \\
\hline & (black) & (grayish brown) & \\
\hline \multirow{2}{*}{6} & $10 Y R 6 / 2$ & 10YR 6/2 & 10YR $2 / 2$ (very dark brown) y \\
\hline & (light brownish gray) & (light brownish gray) & 10YR 3/4 (dark yellowish brown) \\
\hline
\end{tabular}


muestras manifiestan variaciones en la coloración -núcleo algo más oscuro que los bordes o bordes de diversas tonalidades-, lo que indicaría cambios en la atmósfera de cocción (Orton et al. 1997). Asimismo, hay otros factores que pueden influir en la coloración, tales como la composición de las arcillas, el combustible utilizado o la absorción de las sustancias durante el uso, entre otros (Shepard, 1956; Orton et al. 1997; González, 2005).

\section{Análisis microscópicos}

El análisis petrográfico indica variabilidad en la estructura de las pastas. Éstas pueden ser agrupadas en pastas con alto porcentaje de inclusiones $(25 \%$ a $50 \%)$ y con una densidad de poros alta $(n=2)$ o moderada $(n=4)$. La porosidad varía entre $5 \%$ y $30 \%$. La matriz, compuesta por minerales arcillosos, registra valores comprendidos entre 30 y $70 \%$ (Tabla 3). Dos muestras presentan inclusiones minerales orientadas parcialmente en los extremos del corte. Este ordenamiento podría deberse a la técnica de modelado (Rye, 1994; Solá, 2001).

En todas las muestras se reconocen cuarzos y fragmentos líticos. El cuarzo se registra en porcentajes similares $-20 \%$ a $35 \%$-, mientras que los fragmentos líticos se encuentran en porcentajes altamente variables $-5 \%$ a $75 \%$ También la composición de los fragmentos líticos
Tabla 3. Características de la pasta.

\begin{tabular}{|c|c|c|c|}
\hline \multirow[b]{2}{*}{ Muestra } & \multicolumn{3}{|c|}{$\%$} \\
\hline & Inclusiones & Porosidad & Matriz \\
\hline 1 & 40 & 25 & 35 \\
\hline 2 & 50 & 15 & 35 \\
\hline 3 & 25 & 5 & 70 \\
\hline 4 & 40 & 30 & 30 \\
\hline 5 & 40 & 10 & 50 \\
\hline 6 & 40 & 10 & 50 \\
\hline
\end{tabular}

es diversa. En la muestra 1 predominan las rocas ígneas ácidas, registrándose a su vez escasas rocas volcánicas -posiblemente basálticas-, metamórficas y sedimentarias. La muestra 2 registra vulcanitas ácidas ricas en vidrio y alteradas a zeolitas, a la vez que se reconocen escasas rocas volcánicas básicas. La muestra 3 contiene rocas metamórficas y sedimentarias, probablemente areniscas. En la muestra 4 dominan las rocas volcánicas básicas. Asimismo, se reconocen escasas rocas ígneas ácidas. Por último, las muestras 5 y 6 exhiben un predominio de rocas volcánicas ácidas alteradas a arcillas, registrándose también escasas rocas metamórficas. Además, se identifican feldespatos plagioclasas (Muestras 1, 2, 3, 4 y 6) y minerales opacos (Muestras 2, 3, 4, 5 y 6). El primero de los minerales con porcentajes comprendidos entre <

Tabla 4. Porcentaje de inclusiones. Referencias: Hbl (Hornblenda); Ep (Epidota); Px (Piroxeno); AAmp (Anfíbol alcalino, oxihornblenda); Fpla (Feldespato plagioclasa); Fpo (Feldespato potásico); Bt (Biotita); Opq (Minerales opacos); Amp (Anfíbol); Zrn (Circón); Mi (Microtiesto); Otros min (Otros minerales). Las inclusiones con porcentajes $<2 \%$ han sido agrupadas en Otros minerales.

\begin{tabular}{|c|c|c|c|c|c|c|}
\hline \multirow{2}{*}{$\begin{array}{l}\text { Tipo de } \\
\text { Inclusión }\end{array}$} & \multicolumn{6}{|c|}{ Muestra e inclusiones (\%) } \\
\hline & 1 & 2 & 3 & 4 & 5 & 6 \\
\hline Cuarzo & 35 & 20 & 35 & 25 & 30 & 30 \\
\hline Fragmentos líticos & 25 & 75 & 15 & 5 & 55 & 45 \\
\hline Feldespato Plagioclasa & 10 & Otros min & 25 & $<20$ & - & Otros min \\
\hline Feldespato Potásico & 10 & - & 20 & 15 & - & Otros min \\
\hline Biotita & 15 & - & Otros min & 10 & - & 5 \\
\hline Minerales opacos & - & $<5$ & Otros min & 10 & 15 & 15 \\
\hline Anfíbol (hornblenda) & Otros min & - & Otros min & 10 & - & - \\
\hline Piroxeno & Otros min & Otros min & - & 5 & - & Otros min \\
\hline \multirow{2}{*}{ Otros minerales } & 5 & $<1$ & 5 & $<3$ & & 5 \\
\hline & $\mathrm{Hbl}, \mathrm{Ep}, \mathrm{Px}, \mathrm{AAmp}$ & Px, Fpla & Ep, Bt, Opq, Amp & Ep, Zrn, Mi & & Fpo, Fpla, Px,Ep \\
\hline Total & 100 & 100 & 100 & 100 & 100 & 100 \\
\hline
\end{tabular}




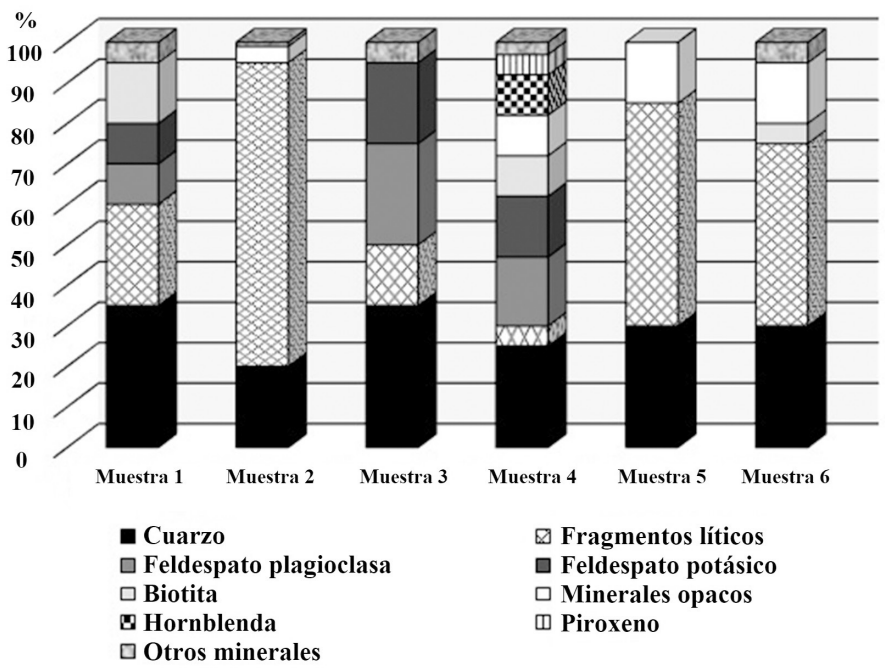

Fig. 3. Porcentaje de las inclusiones en las muestras cerámicas.

1 y 25 , mientras que el segundo, entre < 1 y 15 . Se reconocen inclusiones de feldespato potásico en las muestras $1,3,4$ y $6(<1 \%$ a $20 \%)$, biotita en las muestras $1,3,4$ y $6(<1 \%$ a $15 \%)$, piroxeno en las muestras $1,2,4$ y $6(<1 \%$ a $5 \%)$ y epidota en las muestras $1,3,4$ y $6(<1 \%)$. Se registra anfíbol en las muestras 1,3 y $4(<1 \%$ a $10 \%)$, y circón $(<$ $1 \%$ ) y microtiesto $(<1 \%)$ en la muestra 4 (Tabla 4 ; Fig. 3 y 4). Este último presenta forma angulosa y se distingue al microscopio por la coloración y orientación de sus componentes, los cuales difieren de los registrados en la matriz.

Las inclusiones de un mismo mineral registraron formas diversas entre los tiestos, exceptuando a aquéllas presentes en las muestras 5 y 6 (Tabla 5). Éstas contienen elementos mayoritarios con morfologías semejantes.

Por otra parte, se consignaron los tamaños mínimos y máximos de las inclusiones de los seis tiestos (Tabla 6). Los tamaños más representativos de los granos de las inclusiones mayoritarias permitieron fijar las texturas de las pastas según las dimensiones establecidas para las arenas. En este sentido, se detectaron texturas medianas/finas $(16,67 \%)$, medianas $(66,66 \%)$ y gruesas $(16,67 \%)$.

Los resultados obtenidos apuntan a la variabilidad de los tiestos. Ésta se manifiesta claramente en el color y en los tratamientos de las superficies -tosco, distinto grado de alisado, pulido y falso engobe-- Por otra parte, los análisis petrográficos permitieron identificar variaciones
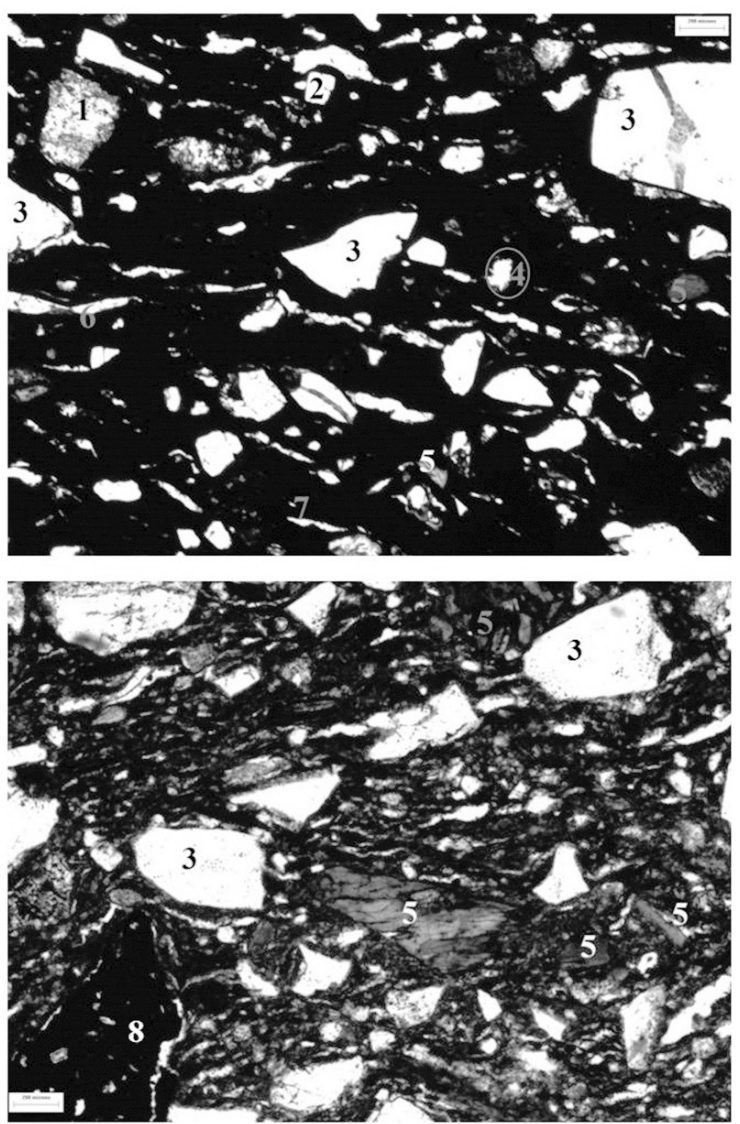

Fig. 4. Microfotografías de las muestras 1 (superior) y 4 (inferior). Referencias: 1: Feldespato potásico levemente alterado a arcilla, 2: Feldespato plagioclasa, 3: Cuarzo, 4: Epidota, 5: Anfíbol (Hornblenda), 6: Biotita, 7: Poro y 8: Microtiesto. 
Tabla 5. Forma de las inclusiones. Referencias: A (anguloso); SA (sub-anguloso); R (redondeado); SR (sub-redondeado).

\begin{tabular}{lcccccc}
\hline \multicolumn{1}{c}{ Forma } & \multicolumn{7}{c}{ Muestra } \\
\cline { 2 - 7 } & 1 & 2 & 3 & 4 & 5 & 6 \\
\hline Cuarzo & A, SA, SR, R & A & A, SA, SR, R & A, SA, SR & A, SA & A, SA \\
Fragmentos líticos & SA & A, SA, SR & SR, R & SR, R & SR, R & SR, R \\
Feldespato Plagioclasa & SR, R & - & SA, SR & A, SR & - & - \\
Feldespato Potásico & SR, R & - & SR & SR & - & - \\
Biotita & A & - & - & SA & - & SA \\
Minerales opacos & - & SR, R & - & SR, R & SR, R & SA, SR, R \\
Anfíbol & - & - & - & A, SA & - & - \\
Piroxeno & - & - & - & SA & - & - \\
Microtiesto & - & - & - & A & - & - \\
Otros & SR & SR & SR & SR & - & SA, SR \\
\hline
\end{tabular}

Tabla 6. Tamaño mínimo y máximo de las inclusiones $(\mathrm{mm})$.

\begin{tabular}{lcccccc}
\hline & \multicolumn{7}{c}{ Muestra } \\
\cline { 2 - 7 } \multicolumn{1}{c}{ Inclusiones } & 1 & 2 & 3 & 4 & 5 & 6 \\
\hline Cuarzo & $0,06-1,8$ & $0,04-0,68$ & $0,05-2$ & $0,02-0,8$ & $0,04-1,2$ & $0,04-0,9$ \\
Fragmentos líticos & $0,5-3,2$ & $0,08-1,4$ & $0,2-1,2$ & $0,4-0,7$ & $0,04-1$ & $0,06-3,6$ \\
Feldespato Plagioclasa & $0,16-0,6$ & - & $0,2-1,2$ & $0,1-0,9$ & - & - \\
Feldespato Potásico & $0,1-0,6$ & - & $0,12-0,8$ & $0,16-0,38$ & - & - \\
Biotita & $0,1-0,7$ & - & - & $0,06-1$ & - & $0,04-0,34$ \\
Minerales opacos & - & $0,02-0,2$ & - & $0,05-0,7$ & $0,04-0,8$ & $0,08-1,5$ \\
Anfíbol & - & - & - & $0,05-0,8$ & - & - \\
Piroxeno & - & - & - & $0,4-0,52$ & - & - \\
Microtiesto & - & - & - & $0,3-0,7$ & - & - \\
Otros & $0,1-0,2$ & $0,17-0,8$ & $0,3-0,8$ & $0,1-0,4$ & - & $0,2-0,9$ \\
\hline
\end{tabular}

en el tipo, porcentaje, forma y tamaño de las inclusiones, lo que podría sugerir el uso de fuentes de materias primas disímiles.

\section{CRONOLOGÍA Y VALORES ISOTÓPICOS DE LOS RESIDUOS ORGÁNICOS}

Los tiestos que brindaron información cronológica proceden de la cuenca media del río Chalía y curso superior del río Santa Cruz. Las sustancias adheridas fechadas por AMS arrojan dataciones muy tardías de $420 \pm 20$ y $140 \pm 20$ años AP (Tabla 7; Edad mediana de probabilidad de 1.484 y 1.857 años DC respectivamente). Las calibraciones realizadas indican que los fechados están posiblemente comprendidos entre momentos inmediatamente previos al contacto hispano-indígena (a partir de 1.520 años DC, $v$. Pigaffeta, 2001 [1899]) y momentos históricos ecuestres (desde 220 años AP o 1.730 años DC, v. Moreno \& Videla, 2008). Estos datos son concordantes con las dataciones obtenidas sobre residuos orgánicos en tiestos recuperados al noroeste y oeste del área de estudio (Cassiodoro \& Tessone, 2014; Chaile et al. 2019) y con aquellas reseñas brindadas por las fuentes documentales que señalan la utilización de la tecnología cerámica durante el primer contacto costero (Pigaffeta, 2001 [1899]) y la primera mitad del siglo XX (Aguerre, 2000). 
Tabla 7. Valores de isótopos y edades de radiocarbono AMS para los residuos orgánicos adheridos a los fragmentos de cerámica.

*Las incertidumbres de edad incluyen un error multiplicador de laboratorio (Stuiver \& Reimer, 1993; Hogg et al. 2013).

\begin{tabular}{|c|c|c|c|c|c|c|}
\hline \multirow[b]{2}{*}{ Muestra } & \multirow[b]{2}{*}{ Raspado } & \multirow[b]{2}{*}{$\delta 15 \mathrm{~N}(\% \circ)$} & \multirow[b]{2}{*}{$\boldsymbol{\delta} 13 \mathrm{C}(\% \circ)$} & \multicolumn{2}{|c|}{ Fechados } & \multirow[b]{2}{*}{ código laboratorio } \\
\hline & & & & $\mathrm{AP}$ & DC (2 sigma) & \\
\hline \multirow[t]{2}{*}{5} & Externo & - & -23 & $420 \pm 20$ & $1451-1507(p=0,759)$ & UGAMS\#15806 \\
\hline & & & & & $1585-1619(p=0,241)$ & \\
\hline \multirow[t]{2}{*}{1} & Interno & 6,2 & $-27,8$ & $140 \pm 20$ & $1806-1950(\mathrm{p}=0,820)^{*}$ & UGAMS\# 15805 \\
\hline & & & & & $1695-1726(p=0,180)$ & \\
\hline
\end{tabular}

Con respecto al análisis del residuo orgánico recuperado en la cara externa de la muestra 5, los datos son poco concluyentes. El residuo proporcionó un valor de $\delta^{13} \mathrm{C}$ de -23\%o, similar a aquéllos obtenidos para plantas $\mathrm{C}_{3}$ de la estepa (Chaile et al. 2018) y carbones arqueológicos de las cuencas de los ríos Chico y Santa Cruz -interior- (Tabla 8). Esto sugiere que el residuo, posiblemente hollín, fue generado por la quema de madera $\mathrm{C}_{3}{ }^{1}$. No obstante, la ausencia de un valor de $\delta^{15} \mathrm{~N}$, no permite obtener resultados claros. Cabe destacar que un $\delta^{13} \mathrm{C}$ de $-23 \%$, acompañado de un valor de $\delta^{15} \mathrm{~N}$ elevado podría también ser interpretado como residuo de proteína animal ( $v$. Cassiodoro \& Tessone, 2014). En contraste, el residuo orgánico obtenido de la cara interna de la muestra 1 produjo valores de $\delta^{13} \mathrm{C}$ y $\delta^{15} \mathrm{~N}$ de $-27,8$ y $6,2 \%$ o, respectivamente (Tabla 7). En vista de estos valores, se considera que podría tratarse de grasa de Lama guanicoe y/o Pterocnemia pennata. La grasa subcutánea y medular de Lama guanicoe tiene valores de $\delta^{13} \mathrm{C}$ de $-30,8$ y $-28,6 \%$ o, respectivamente, y tendría valores de $\delta^{15} \mathrm{~N}$ similares al colágeno óseo, que en guanaco es de aproximadamente $6,3 \%$ y en choique 7,8 \%o (Tabla 8 ). Es probable que el residuo orgánico sea una mezcla de grasa subcutánea y medular con algo de músculo (-21,5 \%o para el músculo guanaco y $-25,5$ \%o para el músculo choique; Tabla 8) para obtener un valor de -27,8 \%o. Además, dada la forma en que la grasa y la proteína se reducen, la grasa de guanaco y choique suele registrar un valor global de $\delta^{13} \mathrm{C}$ más bajo que el músculo o el colágeno (Chaile et al. 2018).

\section{DISCUSIÓN}

En este trabajo se ofreció un marco cronológico para el uso de la tecnología cerámica en el área central de la provincia de Santa Cruz, en espacios intermedios entre la costa y la cordillera. En este sentido, las dataciones se corresponden con el último tramo del Holoceno tardío, entre los siglos XV y XX DC.

La búsqueda de variación en tiestos cerámicos, problemática tan poco explorada en el sur de la región patagónica, permitió detectar diferencias entre las muestras. Los tiestos, tanto por su localización espacial, como por sus cronologías y variabilidad macro-microscópica, pudieron ser atribuidos a un número mínimo de seis ejemplares. Algunas de las diferencias se manifiestan en el color de la pasta y en los tratamientos de las superficies. Si bien ninguno de los ejemplares se encuentra decorado, cabe mencionar que entre los fragmentos cerámicos relevados por Verneau (1903) y Gradin y Aguerre (Gradin, 2000) entre los ríos Chalía y Santa Cruz, se registraron incisiones.

Los datos petrográficos muestran variaciones microscópicas en el tipo, porcentaje, forma y tamaño de las inclusiones que sugerirían distintas áreas de procedencia. También se reconoció variabilidad en la densidad de poros -entre 5\% y 30\%-, aunque Bronitsky (como se citó en González de Bonaveri et al. 2000) señaló que una composición porcentual de poros cercana a los $10-25 \%$ es ideal para que los materiales cerámicos alcancen durabilidad y resistencia. Asimismo, se identificó cierta homogeneidad en el grosor de las paredes -mediano y delgado- $y$ en el porcentaje

1 Estudios experimentales que evalúan los valores $\delta^{13} \mathrm{C}$ en restos vegetales muestran que aquéllos procedentes de sitios arqueológicos pueden estar sometidos a un fraccionamiento y desplazamiento como consecuencia de procesos de combustión y degradación que influyen en la modificación de la señal isotópica (v. Tessone et al. 2010 y citas allí incluidas). 
Tabla 8. Características isotópicas de los residuos obtenidos en la superficie de fragmentos cerámicos y del tejido animal y vegetal de la región esteparia. Todas las muestras son actuales salvo que se indique lo contrario. Para que los datos modernos puedan compararse con los arqueológicos se consideró el efecto Suess y se agregó 1,5 \%o a los valores modernos de $\delta^{13} \mathrm{C}$ (Tessone \& Panarello, 2008-2010; Tessone et al. 2013). Ref. Referencias; 1 . Este trabajo; 2. Chaile et al. (2018); 3. Tessone y Panarello (2008-2010); 4. Brook et al. (2018).

\begin{tabular}{|c|c|c|c|c|c|c|c|c|}
\hline \multirow[b]{2}{*}{ Material } & \multirow[b]{2}{*}{$\mathrm{n}$} & \multicolumn{3}{|c|}{$\begin{array}{c}\boldsymbol{\delta} 13 \mathrm{C} \\
(\% \circ)\end{array}$} & \multicolumn{3}{|c|}{$\boldsymbol{\delta} 15 \mathrm{~N}(\% \circ)$} & \multirow[b]{2}{*}{ Ref. } \\
\hline & & Media & Min & Max & Media & Min & Max & \\
\hline Tiesto - residuo orgánico (río Chalía) & 1 & -23 & - & - & - & - & - & 1 \\
\hline Tiesto - residuo orgánico (río Santa Cruz) & 1 & $-27,8$ & - & - & 6,2 & - & - & 1 \\
\hline Tiesto - residuo orgánico & 11 & $-25,7$ & $-27,9$ & -23 & 7,7 & 4,5 & 11 & 2 \\
\hline Lama guanicoe - grasa subcutánea & 1 & $-30,8$ & - & - & - & - & - & 3 \\
\hline Lama guanicoe - grasa medular & 1 & $-28,6$ & - & - & - & - & - & 3 \\
\hline Lama guanicoe - músculo & 1 & $-21,5$ & - & - & - & - & - & 3 \\
\hline Lama guanicoe - colágeno óseo & 76 & $-19,5$ & - & - & 6,3 & - & - & 2 \\
\hline Lama guanicoe - colágeno óseo & 29 & $-19,7$ & $-18,1$ & $-21,3$ & - & - & - & 3 \\
\hline Lama guanicoe - colágeno óseo & - & - & $-18,6$ & $-22,3$ & - & - & - & 4 \\
\hline Pterocnemia pennata - músculo & 1 & $-25,5$ & - & - & - & - & - & 3 \\
\hline Pterocnemia pennata - colágeno óseo & 35 & $-20,6$ & - & - & 7,8 & - & - & 2 \\
\hline Pterocnemia pennata - colágeno óseo & 11 & $-20,7$ & $-19,7$ & $-22,7$ & - & - & - & 3 \\
\hline Plantas (estepa) & 7 & $-24,3$ & - & - & 3,9 & - & - & 2 \\
\hline Plantas C3 & 18 & $-25,8$ & $-24,3$ & $-28,3$ & - & - & - & 3 \\
\hline Plantas C4 & 1 & -13 & - & - & - & - & - & 3 \\
\hline Carbón arqueológico (río Chico, interior) & - & - & -22 & $-23,7$ & - & - & - & 4 \\
\hline Carbón arqueológico (río Santa Cruz, interior) & - & - & $-21,3$ & $-22,9$ & - & - & - & 4 \\
\hline
\end{tabular}

de inclusiones -alta, aunque con variaciones-. Las similitudes registradas podrían estar vinculadas con una funcionalidad especifica de los recipientes. En este sentido, los porcentajes de porosidad, las dimensiones de los espesores -medianos y delgados- y la presencia de hollin permiten inferir la cocción de alimentos, posiblemente recursos de estepa, según los resultados isotópicos obtenidos. Cabe destacar que la interpretación del residuo orgánico de la muestra 1 como grasa animal coincide con lo que Chaile y colaboradores (2018) interpretaron en otros tiestos de Patagonia. En relación con lo anterior, se menciona que estudios etnográficos e informes de viajeros documentaron también el uso de ollas para preparar grasa animal para el consumo humano" (referencias en Chaile et al. 2018).

Dada la recuperación de fragmentos con bordes mayormente angulosos, se propone el descarte de las piezas por rotura de forma próxima a los lugares de los hallazgos. La baja densidad de tiestos y la variabilidad microscópica se oponen a la producción local de la tecnología cerámica y abogan por su transporte ocasional desde otros espacios, probablemente por grupos con alta movilidad (para más información sobre contextos de producción cerámica $v$. González, 2005). Esto coincidiría con lo sugerido por algunos investigadores quienes concluyeron que la cerámica de Patagonia sur habría sido adquirida por intercambio con otros 
grupos localizados más al norte (Mena \& Jackson, 1991; Martinic \& Prieto, 1998).

Cabe destacar que la baja frecuencia de microtiestos $(<1 \%)$ en uno de los cortes cerámicos -Muestra 4- podría ser resultado de un aporte no intencional. De acuerdo con esto, la inclusión de este material sugeriría muy posiblemente la obtención de arcillas en cercanías de algún sector que contenga fragmentos cerámicos. Por el momento, no se ha identificado esta inclusión entre los tiestos analizados al noroeste del área de estudio, espacio considerado como una posible área de manufactura cerámica ( $v$. Cassiodoro \& Tchilinguirian, 2007). No obstante, se ha reconocido en baja frecuencia en la costa norte de Chubut -sitio La Armonía 2- ( $v$. Schuster, 2014), a una distancia lineal de ca. 850 km -extremo sur del Macizo del Deseado-. Por otra parte, los tiestos recuperados en la cuenca media del río Chico -Muestra 6- y río Chalía -Muestra 5- registran forma y composición de inclusiones semejantes, así como porcentaje de inclusiones, poros y matriz similares. Si bien la muestra es escasa, las semejanzas mencionadas podrían aludir al uso de fuentes de materias primas de constitución semejante y/o a su posible obtención de grupos con modos similares de confeccionar cerámica.

Los escasos tiestos dispersos en el área de estudio se pueden asociar con ocupaciones cortas que limitan las posibilidades de rotura de recipientes y/o a su uso ocasional (e.g. Martinic, 1995; Cornejo \& Sanhueza, 2003). Los estudios arqueológicos realizados entre el extremo sur del Macizo del Deseado y la margen norte de la cuenca del río Santa Cruz apuntan a que se trata de poblaciones muy móviles, para las que la disponibilidad de agua juega un papel importante (e.g. Franco et al. 2014; Brook et al. 2015; Cirigliano, 2016). La situación, en este sentido, contrasta con la observada al NO de la provincia de Santa Cruz, para la que se ha sostenido una baja movilidad residencial y la manufactura local de cerámica (Chaile et al. 2019; entre otros).

\section{CONCLUSIÓN}

Este trabajo brindó las dataciones radiocarbónicas más australes de residuos adheridos a fragmentos cerámicos, situando el uso de esta tecnología a fines del Holoceno tardío. El registro cerámico en el área de estudio es escaso, lo que puede ser asociado con una baja frecuencia en el uso de esta tecnología. La evidencia exigua, sumada a la alta diversidad microscópica que registran los tiestos podrían sugerir que los individuos obtenían recipientes cerámicos de manera ocasional de otros grupos localizados a diferentes distancias del área investigada. Si bien los datos actuales apuntan en ese sentido, la baja frecuencia de tiestos y la existencia de espacios aún no explorados, invitan a revisar y ampliar lo aquí expuesto a la luz de los nuevos hallazgos arqueológicos.

\section{AGRADECIMIENTOS}

Los trabajos fueron financiados con los proyectos PIP (CONICET) 0447, PICT 2015-2038 (ANPCyT) y UBACyT (Universidad de Buenos Aires) 20020130100664BA y 20020170100663BA. La Universidad de Georgia proporcionó fondos para la datación por radiocarbono (AMS) y análisis isotópicos. El trabajo forma parte de una beca de Doctorado CONICET (Cirigliano, 2016). Se agradece a los evaluadores anónimos que con sus comentarios contribuyeron a mejorar el manuscrito original y al Comité Editor por el tiempo invertido en el proceso de publicación. A Annette Aguerre, por su predisposición para brindar información sobre los trabajos previos en el área. A Augusto Tessone por sus comentarios sobre isótopos y a Valeria Palamarczuk, Marisa Kergaravat, Ivana Ozán y Marcelo Vitores por los correspondientes a la muestra cerámica. A Mónica Reinsch de la Ea. La Marta y a Otto Hemlich de la Ea. El Tranquilo por proporcionar tres de las muestras cerámicas aquí analizadas. A Minera Piedra Grande S.A. por brindarnos alojamiento y comida. A Gerardo Povaszán, Otto e Hilde Hemlich, Pablo Ramírez, Marcelo Cebeira y familia Hofmann por su predisposición y valiosa colaboración.

\section{BIBLIOGRAFÍA}

Aguerre, A. M. (1987). Investigaciones arqueológicas en el "área de la Martita", Depto. Magallanes, Prov. Santa Cruz. En Comunicaciones. Primeras Jornadas de Arqueología de la Patagonia (pp. 17-32). Trelew, Argentina: Dirección de Cultura de la Provincia de Chubut. 
Aguerre, A. M. (2000). Las vidas de Pati en la toldería Tehuelche del Río Pinturas y el después. Buenos Aires, Argentina: Universidad de Buenos Aires, Facultad de Filosofía y Letras.

Balfet, H., Fauver-Berthelot, M. F., \& Monzón, S. (1992). Normas para la descripción de vasijas. Ciudad de México, México: Centro de estudios mexicanos y centroamericanos (CEMCA).

Belardi, J. B., Espinosa, S., Barrientos, G., Carballo Marina, F., Re, A., Campan, P., Súnico, A., \& Guichón, F. (2013). Las mesetas de San Adolfo y Cardiel Chico: Estrategias de movilidad y tácticas de caza de guanacos en el SO de Santa Cruz. En A. F. Zangrando, R. Barberena, A. F. Gil, G. A. Neme Gustavo, M. A. Giardina, L. Luna, C. Otaola, S. L. Paulides, L. M. Salgan \& A. M. Tívoli (Comp.), Tendencias teórico-metodológicas y casos de estudio en la Arqueología de Patagonia (pp. 261270). Buenos Aires, Argentina: Altuna Impresores.

Bellelli, C. (1980). La decoración de la cerámica gris incisa de la Patagonia, República Argentina. Revista del Museo Paulista, XXVII, 199-225.

Bird, J. (1988). Travels and Archaeology in South Chile. Iowa, Estados Unidos: University of Iowa Press.

Borrero, L. A. (1994-95). Arqueología de la Patagonia. Palimpsesto. Revista de Arqueología, 4, 9-96.

Braun, D. (1983). Pots as tools. En A. Keene \& J. Moore (Eds.), Archaeological Hammers and Theories (pp. 107134). New York, Estados Unidos: Academic Press.

Brook, G. A., Franco, N. V., Ambrústolo, P., Mancini, M. V., Wang, L., \& Fernández, P. M. (2015). Evidence of the earliest humans in the Southern Deseado Massif (Patagonia, Argentina), Mylodontidae, and change in water availability. Quaternary International, 363, 107-125.

Brook, G. A., Franco, N. V., Cherkinsky, A., Acevedo, A., Fiore, D., Pope, T. R., \& Salguero, T. T. (2018). Pigments, binders, and age of rock art at Viuda Quenzana, Santa Cruz, Patagonia (Argentina). Journal of Archaeological Sciences, Reports, 21, 47-63.

Cassiodoro, G. (2008). La tecnología cerámica en cazadores recolectores del noroeste de la provincia de Santa Cruz. En A. Austral \& M. Tamagnini (Comp.), Problemáticas de la Arqueología Contemporánea, Tomo 2 (227237). Río Cuarto, Argentina: UNRC.

Cassiodoro, G. (2009). Movilidad y uso del espacio de cazadores-recolectores del Holoceno tardío: estudio de la variabilidad del registro tecnológico en distintos ambientes del noroeste de la provincia de Santa Cruz. Arqueología, 15, 235-239.
Cassiodoro, G. (2010). Composición artefactual de los conjuntos de superficie de la cuenca del lago Salitroso (Santa Cruz, Argentina). Magallania, 38(1), 215-236.

Cassiodoro, G., \& Tchilinguirian, P. (2007). Análisis petrográficos de cerámicas en el noroeste de la provincia de Santa Cruz. En F. Morello, M. Martinic, A. Prieto \& G. Bahamonde (Eds.), Arqueología de FuegoPatagonia. Levantando piedras, desenterrando huesos...y develando arcanos (pp. 839-852). Punta Arenas, Chile: CEQUA.

Cassiodoro, G., \& García Guraieb, S. (2009). Análisis del registro tecnológico y osteológico de los entierros humanos del Holoceno tardío del lago Salitroso (Santa Cruz). En M. Salemme, F. Santiago, M. Álvarez, E. Piana, M. Vázquez \& M. Mansur (Comp.), Arqueología de Patagonia: una mirada desde el último confín, Tomo 2 (pp. 613-628). Ushuaia, Argentina: Editorial Utopías.

Cassiodoro, G. A., \& Tessone, A. (2014). Análisis radiocarbónico y de isótopos estables en residuos cerámicos del centrooeste de Santa Cruz (Patagonia). Relaciones de la Sociedad Argentina de Antropologia, XXXIX(1), 293-299.

Cassiodoro, G., Flores Coni, J. \& Dellepiane, J. (2013). Cronología y asentamiento en la meseta del Guitarra (Santa Cruz): El sitio Cañadón Guitarra 3. En A. F. Zangrando, R. Barberena, A. F. Gil, G. A. Neme Gustavo, M. A. Giardina, L. Luna, C. Otaola, S. L. Paulides, L. M. Salgan \& A. M. Tívoli (Comp.), Tendencias teórico-metodológicas y casos de estudio en la Arqueología de Patagonia (pp. 279-306). Buenos Aires, Argentina: Altuna Impresores.

Cassiodoro, G., Espinosa, S., Re, A., Belardi, J. B., Nuevo Delaunay, A., Piriz, F., \& Durou, G. (2014). Tecnología de la cuenca del lago Cardiel. En R. A. Goñi, J. B. Belardi, G. Cassiodoro \& A. Re (Eds.), Arqueología de las cuencas de los lagos Cardiel y Strobel. Poblamiento Humano y Paleoambientes en Patagonia (pp. 67-95). Buenos Aires, Argentina: Aspha.

Chaile, C., Tessone, A., Cassiodoro, G., Bellelli, C., \& Belardi, J. B. (2018). $\delta 13 \mathrm{C}$ and $\delta 15 \mathrm{~N}$ in organic residues of Patagonia pottery. Implications for studies of diet and subsistence strategies among late Holocene huntergatherers. Journal of Archaeological Science: Reports, 20, 47-56.

Chaile, C., Goñi, R., \& Cassiodoro, G. (2019). La cerámica del fin del mundo: el uso de contenedores cerámicos en el centro-oeste de Santa Cruz (Argentina). En A. Laguens, M. Bonnin \& B. Marconetto (Comp.), Libro de 
Resúmenes XX Congreso Nacional de Arqueología Argentina (pp. 1515-1519). Córdoba, Argentina: IDACOR-UNC.

Ciampagna, M. L. (2016). Prácticas en la gestión de recursos vegetales silvestres de grupos cazadores recolectores en los sitios Cormorán Quemado y Nido del Águila, costa norte de Santa Cruz, Patagonia Argentina. En F. Mena (Ed.), Arqueología de la Patagonia: de mar a mar (pp. 345-354). Santiago, Chile: CIEP y Ñire Negro Ediciones.

Cirigliano, N. A. (2016). Movilidad de grupos indígenas $y$ aprovechamiento de materias primas entre el extremo sur del Macizo del Deseado y la cuenca del río Santa Cruz durante los últimos 2.000 años (Provincia de Santa Cruz, Argentina) (Tesis doctoral). Universidad de Buenos Aires, Facultad de Filosofía y Letras, Argentina.

Convención Nacional de Antropología (1966). Primera Convención Nacional de Antropología, Primera parte. Córdoba, Argentina: Universidad Nacional de Córdoba.

Cornejo, L., \& Sanhueza, L. (2003). Coexistencia de cazadores recolectores y horticultores tempranos en la cordillera Andina de Chile Central. Latin American Antiquity, 14(4), 389-407.

Durán, V. A. (1985). Arte rupestre de los cazadores patagónicos en "El Verano", área de La Martita, Departamento Magallanes, Provincia de Santa Cruz. Anales de Arqueología y Etnología, 38/40, 43-75.

Eerkens, J. W. (2003). Residential mobility and pottery use in the Western Great Basin. Current Anthropology, 44 (5), 728-738.

Eerkens, J. W. (2008). Nomadic potters: relationships between ceramic technologies and mobility strategies. En $\mathrm{H}$. Barnard \& W. Wendrich (Eds.), The Archaeology of Mobility: Old World and New World Nomadism (pp307-326). Los Angeles, Argentina: Cotsen Institute of Archaeology.

Eerkens, J. W., Neff, H., \& Glascock, M. D. (2002). Ceramic production among Small-Scale and Movile Hunters and Gatherers: A Case Study from the Southwestern Great Basin. Journal of Anthropological Archaeology, 21, 200-229.

Folk, R. L. (1974). Petrology of Sedimentary Rocks. Austin, Estados Unidos: Hemphill Publishing Co.

Franco, N. V., Cirigliano, N., Ocampo, M., Fiore, D., \& Acevedo, A. (2014). Las ocupaciones del Holoceno tardío en los cañadones basálticos del norte del río Santa Cruz (Patagonia, Argentina). Intersecciones en
Antropología, 15, 377-389.

Frére, M. M., González, M. I., Chan, D., \& Flores, M. C. (2012). Petrografía de la alfarería arqueológica del río Salado Bonaerense. Comechingonia Virtual, 16(2), 115-137.

García, L. C. (1987). Apéndice II -Ficha descriptiva de la cerámica de Las Cuevas 2. En Comunicaciones. Primeras Jornadas de Arqueología de la Patagonia (p. 178). Trelew, Argentina: Dirección de Cultura de la Provincia de Chubut.

Giusta, M. N. (2017). La alfarería del primer milenio AD de la Quebrada de Amaicha (Departamento Tafí del Valle, provincia de Tucumán): aportes sobre sus aspectos tecnológicos y potenciales materias primas. Arqueología, 23(2), 35-61.

González, M. I. (2005). Arqueología de alfareros, cazadores y pescadores pampeanos. Buenos Aires, Argentina: Colección de Tesis Doctorales - SAA.

González de Bonaveri, M., I., Frére, M. M., \& Solá, P. (2000). Petrografía de cerámicas arqueológicas en la cuenca del río Salado, provincia de Buenos Aires. Relaciones de la Sociedad Argentina de Antropología, XXV, 207226.

Goñi, R. A. (2000). Arqueología de momentos históricos fuera de los centros de conquista y colonización: un análisis de caso en el sur de la Patagonia. En J. B. Belardi, F. Carballo Marina \& S. Espinosa (Eds.), Desde el País de los Gigantes. Perspectivas arqueológicas en Patagonia, Tomo I (pp. 283-293). Río Gallegos, Argentina: UNPA.

Goñi, R. A. (2000-2002). Fechados radiocarbónicos y registro arqueológico en la cuenca de los lagos Salitroso/Posadas (Santa Cruz). Cuadernos del Instituto Nacional de Antropología y Pensamiento Latinoamericano, 19, 666-668.

Goñi, R. A., Barrientos, G., \& Cassiodoro, G. (2000-2002). Condiciones previas a la extinción de las poblaciones humanas del sur de Patagonia: una discusión a partir del análisis del registro arqueológico de la cuenca del lago Salitroso. Cuadernos del Instituto Nacional de Antropología y Pensamiento Latinoamericano, 19, 249-266.

Goñi, R. A., Cassiodoro, G., Re, R., Guichón, F., Flores Coni, J., \& Dellepiane, J. (2010). Arqueología de la Meseta del lago Guitarra (Santa Cruz). En R. Bárcena \& H. Chiavazza (Eds.), Arqueología Argentina en el Bicentenario de la Revolución de Mayo, XVII Congreso Nacional de Arqueología Argentina, tomo V (pp. 1923-1928). Mendoza, Argentina: Zeta Editores. 
Goñi, R. A., Cassiodoro, G., \& Rindel, D. (2011-2012). Poblamiento de mesetas: arqueología de Pampa del Asador y Cerro Pampa (Patagonia meridional). Cuadernos del Instituto Nacional de Antropología y Pensamiento Latinoamericano, 23, 21-36.

Gradin, C. J. (1976). Parapetos de piedra y grabados rupestres de la meseta del lago Buenos Aires. Revista del Museo de Historia Natural de San Rafael, II (1/4), 315-337.

Gradin, C. J. (2000). Más allá y más acá del Río Santa Cruz. Buenos Aires, Argentina: Nuevo Offset.

Gradin, C. J., \& Aguerre, A. M. (1983). Arte rupestre del "Área La Martita" sección A del Departamento Magallanes Provincia de Santa Cruz. Relaciones de la Sociedad Argentina de Antropología, XV, 195-223.

Gradin, C. J., \& Aguerre, A. M. (1991). Ocupaciones Patagonienses de la Capa 3 del Alero Cárdenas (Área Río Pinturas), Provincia de Santa Cruz. Arqueología, 1, 197-205.

Gradin, C. J., Aschero, C. A., \& Aguerre, A. M. (1979). Arqueología del Área Río Pinturas (Pcia. Santa Cruz). Relaciones de la Sociedad Argentina de Antropología, XIII, 183-221.

Hammond, H., \& Zubimendi, M. A. (2013). Estudio de la composición de sitios concheros en la Costa Norte de Santa Cruz (Patagonia Argentina). En A. F. Zangrando, R. Barberena, A. F. Gil, G. A. Neme Gustavo, M. A. Giardina, L. Luna, C. Otaola, S. L. Paulides, L. M. Salgan \& A. M. Tívoli (Comp.), Tendencias teóricometodológicas y casos de estudio en la Arqueología de Patagonia (pp. 405-415). Buenos Aires, Argentina: Altuna Impresores.

Hammond, H., Zubimendi, M. A., \& Zilio, L. (2013). Composición de concheros y uso del espacio: aproximaciones al paisaje arqueológico costero en Punta Medanosa. Anuario de Arqueología, 5, 6784.

Hatcher, J. B. (2003 [1903]). Cazadores de huesos en la Patagonia. Expediciones de la Universidad de Princeton a la Patagonia. Marzo 1896-septiembre 1899. Ushuaia: Zagier \& Urruty Publications.

Hogg, A. G., Hua, Q., Blackwell, P. G., Buck, C. E., Guildersno, T. P., Heaton, T. J., \& Zimmerman, S. R. H. (2013). SHCal13 Southern Hemisphere Calibration, 0-50.000 years cal BP. Radiocarbon, 55(4), 1889-1903.

Instituto Geográfico Nacional de la República Argentina. (2018). Capas SIG. Recuperado de http://www.ign. gob.ar/NuestrasActividades/InformacionGeoespacial/ CapasSIG

Kingery, W. D., Bowen, H. K., \& Uhlmann, D. R. (1976).
Introduction to Ceramics. New York, Estados Unidos: John Wiley \& Sons.

Kuhn, S. (1994). A formal approach to the design and assembly of mobile toolkits. American Antiquity, 59(3), 426442.

Lista, R. (2007 [1879]). Viaje al país de los tehuelches. Buenos Aires, Argentina: Patagonia Sur Libros.

Martinic B., M. (1995). Los Aónikenk, historia y cultura. Punta Arenas, Chile: Ediciones Universidad de Magallanes.

Martinic B., M., \& Prieto, A. I. (1998). La cerámica entre los Aonikenk. Anales del Instituto de la Patagonia, 26, 77-82.

Massone, M. (1984). Los paraderos tehuelches y prototehuelches en la costa del estrecho de Magallanes. Anales del Instituto de la Patagonia, 15, 27-42.

Mena, F. L., \& Jackson, D. S. (1991). Tecnología y subsistencia en Alero Entrada Baker Región de Aisén, Chile. Anales del Instituto de la Patagonia, 20, 169-203.

Mena, F., \& Lucero, V. (2004). En torno a las últimas poblaciones indígenas de la cordillera centro-patagónica. Estudio comparado de tres valles de Aysén oriental (Chile). En M. T. Civalero, P. M. Fernández \& A. G. Guráieb (Comp.), Contra viento y marea, arqueología de la Patagonia (pp. 643-657). Buenos Aires, Argentina: INAPL.

Menghin, O. F. A. (1952). Fundamentos cronológicos de la prehistoria de Patagonia. Runa, 5, 23-43.

Mengoni Goñalons, G. (1987). Investigaciones Arqueológicas en el noroeste de la meseta central de Santa Cruz. En Comunicaciones. Primeras Jornadas de Arqueología de la Patagonia (pp. 171-175). Trelew, Argentina: Dirección de Cultura de la Provincia de Chubut.

Moreno, E. J. (2009). Arqueología y etnohistoria de la Costa Patagónica Central en el Holoceno Tardio. Rawson, Argentina: Fondo Editorial Provincial y Secretaría de Cultura del Chubut.

Moreno, E. J., \& Videla, B. A. (2008). Rastreando ausencias: la hipótesis del abandono del uso de los recursos marinos en el momento ecuestre en la Patagonia continental. Magallania, 36(2), 91-104.

Munsell Soil Color Charts (1992). Kew, Australia: Macbeth -Division of Kollmorgen Instruments Corp.

Narborough, J. (2007 [1694]). El viaje de Sir John Narborough al Mar del Sur. En R. C. Paredes, A. Winogrand \& R. Winogrand (Eds.), Un relato de diversos viajes y descubrimientos recientes, Colección Reservada del Museo del fin del mundo (73-165). Buenos Aires, Argentina: Eudeba.

Orton, C., Tyers, P., \& Vince, A. (1997). La Cerámica en 
Arqueología. Barcelona, España: Crítica.

Outes, F. (1904). La alfarería indígena de Patagonia. Anales del Museo Nacional de Buenos Aires, 11(4), 33-41.

Pigaffeta, A. (2001 [1899]). Primer Viaje Alrededor del Mundo. Buenos Aires, Argentina: El Elefante Blanco.

Powers, M. C. (1953). A new roundness scale for sedimentary particles. Journal of Sedimentary Petrology, 23, 117119.

Rice, P. M. (1987). Pottery Analysis: A Sourcebook. Chicago, Estados Unidos: University of Chicago Press.

Roumec, A., Zubimendi, M., \& Ciampagna, L. (2017). Primera caracterización de tiestos cerámicos provenientes de la costa norte de Santa Cruz. En P. Gertsmayer (Ed.), Libro de Resúmenes VII Jornadas de Jóvenes Investigadores y Extensionistas (p. 101). La Plata. Argentina: Museo de La Plata.

Rye, O. S. (1994). Pottery technology: Principles and reconstruction. Washington DC, Estados Unidos: Taraxacum.

Sacchi, M. (2013). A un paso de la laguna: Análisis lítico del sitio Bajo de la Laguna 2, provincia de Santa Cruz. Comechingonia virtual, VII(2), 216-233.

Schuster, V. (2014). La organización tecnológica de la cerámica de cazadores-recolectores. Costa norte de la provincia del Chubut (Patagonia Argentina). Relaciones de la Sociedad Argentina de Antropología, XXXIX(1), 203-231.

Shepard, A. (1956). Ceramics for Archaeologist. Washington DC, Estados Unidos: Carnegie Institution Publications.

Simms, S. R., Bright, J. R., \& Ugan, A. (1997). Plain-ware ceramics and residential mobility: A case study from the Great Basin. Journal of Archaeological Science, 24, 779-92.

Skibo, J. M. (1987). Fluvial sherd abrasion and the interpretation of surface remains. Southwestern North American Archaeologist, 8, 125-142.

Solá, P. (2001). Alcances del estudio petrográfico y análisis petrológico de cerámicas arqueológicas. Informe recuperado de la cátedra de Ergología y Tecnología, Universidad de Buenos Aires, Facultad de Filosofía y Letras.

Solá, P. (2011). Tiestos trazadores de cerámica exótica en sitios tardíos de los alrededores de Susques (Jujuy, Puna argentina). Intersecciones en Antropología, 12, 177194.

Stuiver, M., \& Reimer, P. J. (1993). Radiocarbon Calibration Program. Radiocarbon, 35, 215-230.

Sugrañes, N. (2010). Tecnología cerámica y estrategias de movilidad entre cazadores-recolectores de altura. El caso del sitio Valle Hermoso 1 (Malargüe, Mendoza). Intersecciones en Antropología, 12, 293-304.

Tessone, A., \& Panarello, H. (2008-2010). Isótopos estables del carbono en el centro-noroeste de Santa Cruz (Patagonia, República Argentina): hacia una reconstrucción de la ecología isotópica. Cuadernos del Instituto Nacional de Antropología y Pensamiento Latinoamericano, 22, 217-225.

Tessone, A., Samec, C., Killian Galván, V., \& Panarello, H. (2010). Restos vegetales de origen arqueológico e isótopos estables del carbón: su posibilidad de uso en las reconstrucciones paleodietarias y paleoclimatológicas. En S. Bertolino, R. Cattáneo \& A. D. Izeta (Eds.), La Arqueometría en Argentina y Latinoamérica (pp. 251-256). Córdoba, Argentina: Universidad Nacional de Córdoba.

Tessone, A., Rindel, D., Belardi, J. B., Panarello, H. O., \& Goñi, R. A. (2013). $\delta^{13} \mathrm{C}$ and $\delta^{15} \mathrm{~N}$ variability in modern guanaco (Lama guanicoe) assemblages in Southern Patagonia: Implications for zooarchaeological studies. International Journal of Osteoarchaeology, 24(2), 202-218.

Trola, V., \& Ciampagna, L. (2011). Primeros análisis de tiestos de cerámica en la Costa Norte de Santa Cruz. En A. F. Zangrando, R. Barberena, A. Gil \& G. Neme. (Eds.), Libro de Resúmenes de las VIII Jornadas de Arqueología de la Patagonia (p. 78). San Rafael, Argentina: Museo de Historia Natural de San Rafael.

Verneau, R. (1903). Les anciens patagons. Contribution a l'etude des races précolombienes de l'Amerique du Sud. Monaco: Institut de Paléontologie Humaine.

Vogel, J. S., Southon, J. R., Nelson, D. E., \& Brown, T. A. (1984). Performance of catalytically condensed carbon for use in accelerator mass spectrometry. Nuclear Instruments and Methods in Physics Research Section B, 5(2), 289-293.

Waters, M. R. (1992). Principles of Geoarchaeology. A North American perspective. Arizona, Estados Unidos: The University of Arizona Press.

Zubimendi, M. A. (2015). Distributional archaeology in central San Jorge golf sector (Santa Cruz Province, Patagonia, Argentina). Quaternary International, 373, 104-116.

Zubimendi, M. A., Ambrústolo, P., Zilio, L., \& Castro, A. (2015). Continuity and discontinuity in the human use of the north coast of Santa Cruz (Patagonia Argentina) through its radiocarbon record. Quaternary International, 356, 127-146. 